\title{
AN INTENSE WAVE/PARTICLE EVENT IN THE AURORAL IONOSPHERE
}

\author{
A. D. Johnstone ${ }^{\dagger}$, J. J. Sojkat*, W. Gibbons $\Delta$, B. K. Madahar $\Delta *$, L. J. C. Woolliscroft $\Delta$,
}

† Mullard Space Science Laboratory, University College London, Department of Physics and Astronomy, Holmbury St. Mary, Dorking, Surrey, England

$\Delta$ Department of Physics, University of Sheffield, England

Abstract. An intense burst of VLF waves, at abuencies just above the local proton gyrofrequency, was observed shortly after each of two intense bursts of field-aligned suprathermal electrons $(E<250 \mathrm{eV})$ by instruments carried on a sounding rocket flown in diffuse aurora. If the two phenomena are associated with each other, the implication is that the electron acceleration occurred nearby, in a relatively small volume.

\section{Introduction}

There have been many observations of suprathermal $(5 \mathrm{eV}<\mathrm{E}<500 \mathrm{eV})$ electron bursts in the auroral ionosphere (Raitt and Sojka 1977, Wilhelm 1978). Briefly their characteristics are (1) an increase in suprathermal electron fluxes by more than an order of magnitude (2) strong field-alignment over a wide energy range, but with an increase in intensity at all pitch angles, and (3) a duration of a few seconds in the rocket frame of reference.

We report here the observation of bursts of ELF/VLF waves associated with suprathermal electron bursts. The delay of a few seconds between the particles and the waves is probably caused by velocity dispersion in travelling from the interaction region.

\section{Instrumentation}

The observations were made from a Skylark 12 rocket which reached an apogee of $780 \mathrm{~km}$ after launch from the Andoya Rocket Range at 2159 UT on 0ct.13th 1977. The electron fluxes were measured by a set of four hemispherical electrostatic energy analysers with channel multiplier detectors viewing at angles of $15^{\circ}, 65^{\circ}, 115^{\circ}, 165^{\circ}$ to the spin axis of the rocket. The energy range from $5 \mathrm{eV}$ to $500 \mathrm{eV}$ was covered in 64 logarithmically-spaced steps taking $524 \mathrm{~ms}$ to sweep up and then down. Electron and positive ion fluxes were measured on alternate pairs of sweeps. Every eighth cycle an electron-retarding potential of 18 volts was applied to a grid in the collimator to enable the effects of secondary electron production by high energy electrons inside the analyser to be measured. The effect can be seen regularly every eighth sweep in the electron spectrum below $20 \mathrm{eV}$ in Figure 1 which shows simultaneous electron and wave spectrograms for the period of interest.

\section{*Now at: Utah State University, Logan, TT 84322, USA. \\ **Now at: Dept. of Environmental Sciences, niversity of Lancaster, Lancaster, England.}

Copyright 1981 by the American Geophysical Union.
The spin period of the rocket was $550 \mathrm{~ms}$. It was coning with a half-angle of $7^{\circ}$ about a direction $19^{\circ}$ from the magnetic field direction with a period of $6.6 \mathrm{~s}$. Since the angle of acceptance of the analyser is $t 9^{\circ}$ (full-width) the detectors covered the full angular distribution within a half-rotation of the rocket during most of the flight. The arrangement did not provide a rapid scan of pitch angles at all energies because the spin period is close to the period of the energy cycle which causes a beating effect between energy and pitch angle coverage. However, the pitch angle at a given energy on the downsweep was usually different from the pitch angle at the same energy on the preceding upsweep. Eight point pitch angle distributions were obtained from the four detectors at each energy, once every energy cycle.

Four sensors received the VLF wavefield at the rocket (Gibbons et al., 1980); a double probe electric sensor with a probe separation of $3.96 \mathrm{~m}$ perpendicular to the spin axis, magnetic sensors parallel and perpendicular to the spin axis and a Langmuir probe mounted at the front of the payload. The Langmuir probe detected the VLF waves as an a.c. modulation of the current collected by the probe. The four signals were passed through band pass filters with lower $3 \mathrm{~dB}$ limits at $200 \mathrm{~Hz}$ and upper limits at $20 \mathrm{kHz}$ for the electric sensor, $5 \mathrm{kHz}$ for the magnetic sensor, and $2 \mathrm{kHz}$ for the Langmuir probe and then transmitted to the ground. Spectral analysis and other analogue processing was then carried out subsequently in the laboratory.

\section{Observations}

The flight took place during the recovery phase of an auroral substorm when the aurora, as seen from the launch site, was widespread and diffuse. In Figure 1 the wave frequency/time spectrogram shows a band of noise lying between the local proton gyrofrequency $(f=580 \mathrm{~Hz})$ and $1 \mathrm{kHz}$ with the maximum undally near ${ }^{\mathrm{Ci}} 850 \mathrm{~Hz}\left(1.47 \mathrm{f}_{\mathrm{ci}}\right)$. This noise band was detected at various times throughout the flight but was absent between $220 \mathrm{~s}$ and 300 s when there were no suprathermal electron bursts.

Between 430 s and 460 s there are three bursts of wave noise in this band, with the first two being much stronger than the third (see also Fig. 3). There may be another weaker wave burst earlier in Fig. 3 which is not so obvious in Fig. 1. Its spectrum is narrower in frequency and less structured. The spacing of the bursts is not related to any temporal feature of the instrumentation.

$0^{\text {The electron energy spectrograms are for the }}$ $15^{\circ}$ detector (precipitating, field-aligned electrons) and the $165^{\circ}$ detector (upgoing, 

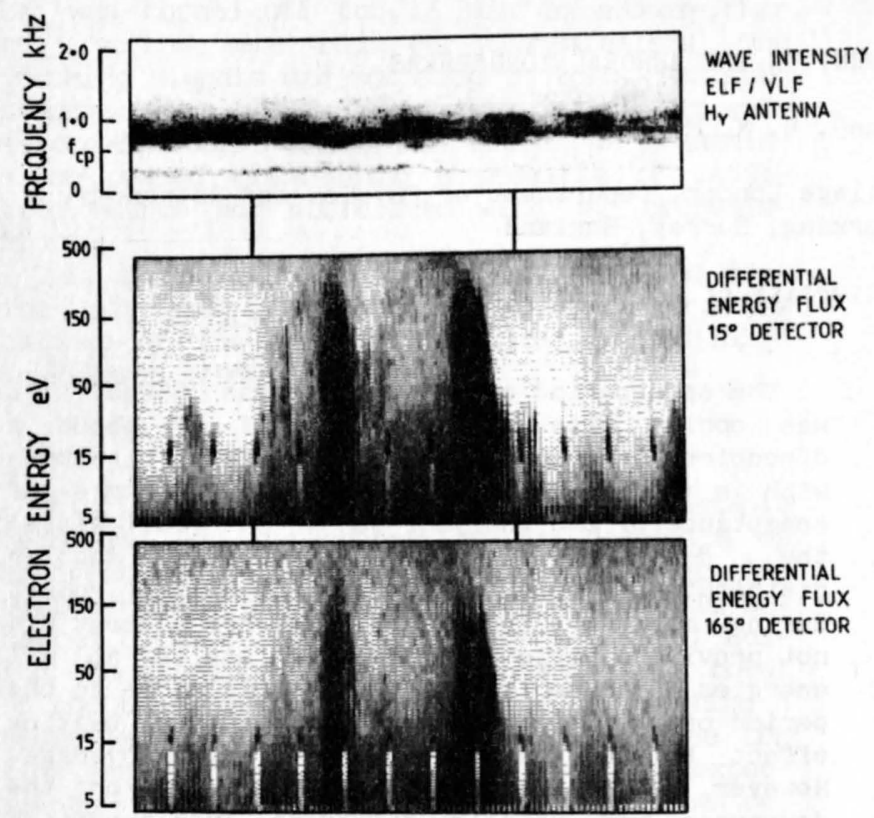

DIFFERENTIAL

ENERGY FLUX $15^{\circ}$ DETECTOR

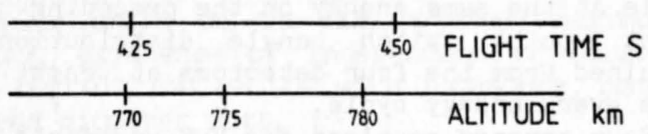

Fig. 1. Wave and electron intensity spectrograms for the suprathermal bursts. The blackness is proportional to the wave or electron intensity. The electron intensity is scaled logarithmically, with black/white being a factor of 40 .

field-aligned electrons). They show two intense field-aligned bursts starting at $431 \mathrm{~s}$ and $444 \mathrm{~s}$. The intensity increases by a factor of 30 at energies up to $250 \mathrm{eV}$ in the downcoming flux and by a factor of 7 up to $150 \mathrm{eV}$ in the upgoing flux. Upsweeps and downsweeps, which occur at different pitch angles have been plotted separately, giving the spectrogram a striped appearance when the distribution is anisotropic. In the precipitating electrons of the burst the stripes are caused by the strong field-alignment. For example, at the beginning of the second burst at $445 \mathrm{~s}$, at an energy of $50 \mathrm{eV}$ on the upsweep the pitch angle is $28^{\circ}$ while on the following downsweep at the same energy it is only $5^{\circ}$ with the intensity a factor of 6 higher. The upflux, in the $165^{\circ}$ detector, is not strongly field-aligned.

Both the downcoming and the upgoing fluxes have a sharp upper energy limit to the burst spectrum which varies in a systematic way through the event. In the $15^{\circ}$ detector this limit starts at $250 \mathrm{eV}$ and gradually decreases to $25 \mathrm{eV}$ in both events. The limit is always lower in the upcoming fluxes, starting at $150 \mathrm{eV}$ and decreasing in parallel with the precipitating electron spectrum to less than $25 \mathrm{eV}$. In both events there is a peak in the intensity spectrum just below the upper energy limit.

We attribute the upgoing fluxes, together with similar fluxes observed at pitch angles between $40^{\circ}$ and $140^{\circ}$ (by two detectors whose data are not presented here) to a local (i.e. in the neighbourhood of the rocket) interaction between the precipitating field-aligned electron beam and the ambient ionosphere (Johnstone and Sojka 1980).

The pitch angle distribution cannot be measure directly, as already explained, but in order to obtain some idea of the field-alignment Fig. 2 has been produced. It is a scatterplot of the ratio $\mathrm{R}(\mathrm{a})$ against $\mathrm{a}$.

$$
R(a)=I_{15}(E) / I_{65}(E)
$$

where $I_{x}(E)$ is the intensity measured by the detector viewing at $x$ deg to the spin axis, at energy $E$ and $a$ is the pitch angle of the $15^{\circ}$ detector. The pitch angle of the $65^{\circ}$ detector ranges from $40^{\circ}$ to $90^{\circ}$ where the electron distribution over the entire energy range is isotropic.

Taking into account the width of the detector angle of acceptance, the figure shows that the burst fluxes are confined within $20^{\circ}$ of the magnetic field direction, and that the degree of field-alignment is the same at all energies.

Figure 3 shows the temporal relationship between the electron bursts and the wave bursts. The electron bursts have been associated with the most intense wave bursts. The wave bursts have the same duration as the electron bursts, i.e. $3.2 \mathrm{~s}$ for the first and $4.4 \mathrm{~s}$ for the second burst within the accuracy allowed by the sampling rate. The wave bursts are also delayed with respect to the electrons, by $8.8 \mathrm{~s}$ in the first case and $4.4 \mathrm{~s}$ in the second.

\section{Discussion}

The variation of the upper energy limit and the peak energy, which both decrease with time, could be caused by velocity dispersion following simultaneous acceleration of all the electrons at some remote location. If so, then the acceleration must have occurred at a distance of

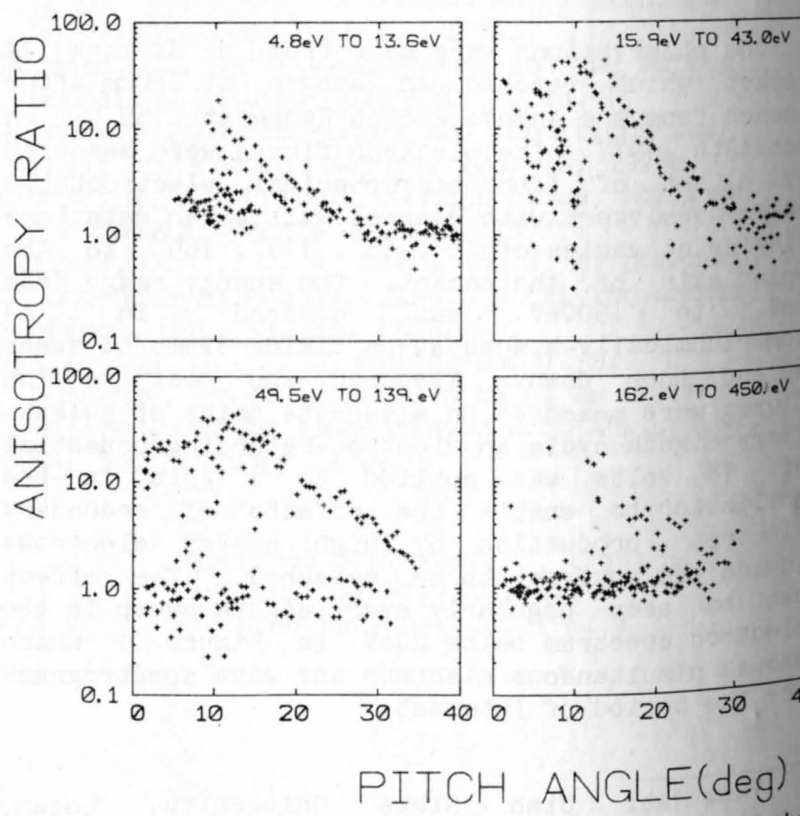

Fig. 2. The anisotropy ratio $R(a)$ sorted into energy intervals for all samples obtained during the second burst $442 \mathrm{~s}$ to $446 \mathrm{~s}$. The e-folding angle for maximum anisotropy is $7^{\circ} \pm 1^{\circ}$ in each interval. 
the order of $6000 \mathrm{kms}$. While this interpretation cannot be eliminated conclusively there are features of the data which make it improbable. The variation of peak energy with time does not follow the curve expected for velocity dispersion from a single source (Smith et al., 1980). Therefore, if there is velocity dispersion, different energy electrons were accelerated in different places. The time profile of the burst (e.g. Fig. 3) at various energies is not just shifted in phase according to velocity but has a different shape. This means that the time profile of the acceleration varies with energy. Finally, a detailed examination of the first burst shows that the peak energy first appears at an energy of $50 \mathrm{eV}$, well below the level reached in the next sweep. If the burst had been generated at a remote location the highest energies would have arrived first. The two bursts have very similar time profiles which suggest that it is a temporal rather than a spatial variation.

Thus we feel that the interpretation of the data most likely to be correct is that the acceleration occurred close enough that velocity dispersion was negligible and that the time variation of the spectrum represents the temporal variation in the source.

The main question is whether the electron bursts and the wave bursts are associated causally. The points in favour of an association are: 1) both the wave and particle events are the most intense of their kind during the flight and occur closely associated in time. The electron angular distributions are the most strongly field-aligned observed during the flight; 2) the duration of the wave bursts is the same as the duration of the associated electron bursts; 3) the general level of the wave noise is low when there are very few bursts.

Points against the association are: 1) there are three wave bursts but only two electron bursts; 2) the three wave bursts are evenly

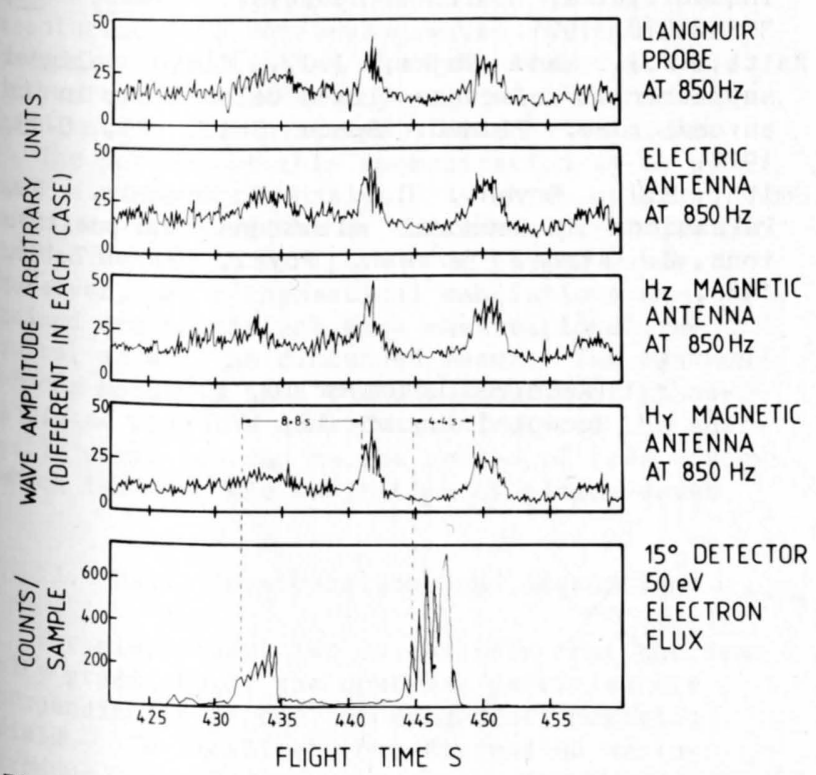

Fig. 3. The time profiles of wave and electron intensities. The latter shows the effect of field-alignment.

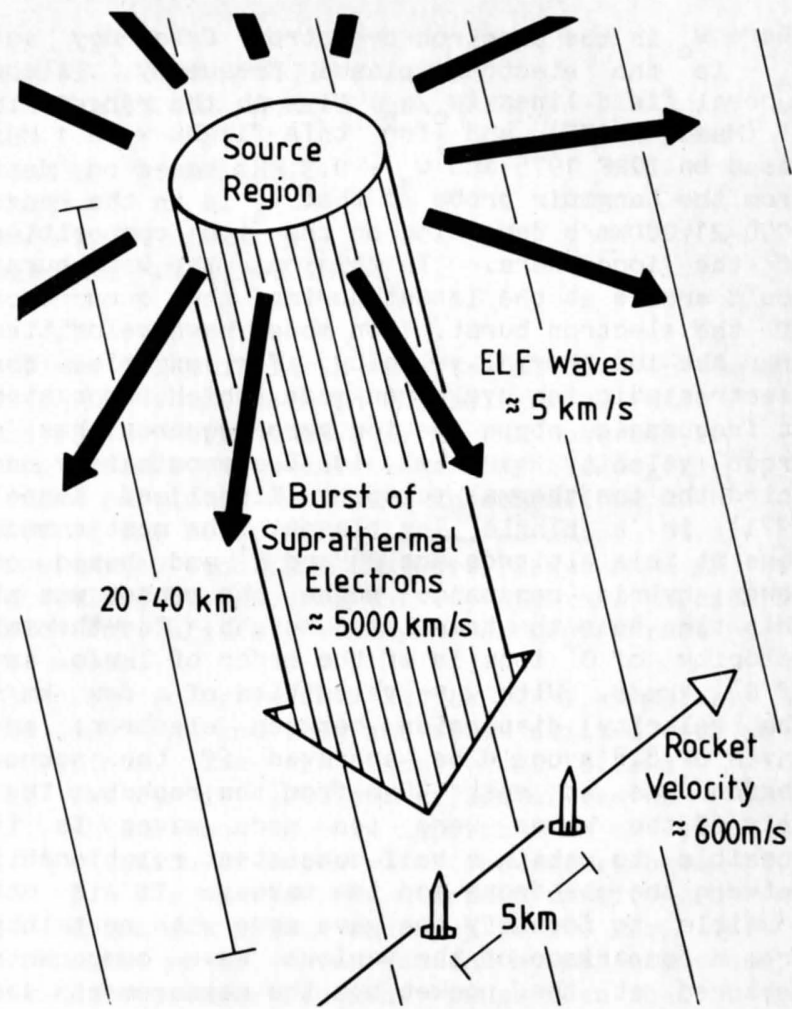

Fig. 4. A possible geometrical relationship for the source region and the observations from the rocket.

spaced in time but the interval between them is different from that between the two electron bursts; 3) other electron bursts detected by this rocket do not have associated wave bursts. No other wave bursts as strong were observed.

There is no way to confirm, with the data, whether the two phenomena are associated physically. If they are not, then we simply have two independent and, as yet, unexplained phenomena to account for. If they are, then the consequences are interesting and lead naturally to plausible explanations for the points against the association. A striking feature of the data is the delay between electrons and waves. It is unlikely to be the result of a spatial separation since that would require the waves to be as closely confined spatially as the electrons, but on different field lines. It is likely that the delay is due to velocity dispersion. The electron velocities are in the range $2900 \mathrm{~km} / \mathrm{s}$ to $9400 \mathrm{~km} / \mathrm{s}$ $(25 \mathrm{eV}$ to $250 \mathrm{eV})$. The wave velocity depends on the mode, which has not yet identified. Three possible characteristic propagation velocities for waves in the frequency range are the whistler mode velocity, the Alfven velocity and the ion thermal velocity. The whistler propagation time to the equator is of the order of 1 sec so that in this case the wave velocity exceeds the maximum electron velocity. If the waves were propagating at this speed they would arrive before the electrons. The Alfven velocity $V_{a}$ in a proton ionosphere is approximately

$$
v_{a}=7000\left(w_{c} / w_{e}\right) \mathrm{km} / \mathrm{s}
$$


where $\mathrm{w}_{\mathrm{c}}$ is the electron cyclotron frequency and $w$ is the electron plasma frequency. Along auroral field lines $\left(w_{c} / w_{e}\right)$ lies in the range 1 to 3 (Maeda 1975) and for this flight $\mathrm{w}_{\mathrm{c}}=1 \mathrm{MHz}$ based on IGRF 1975 and $\mathrm{w}_{\mathrm{H}}=0.3 \mathrm{MHz}$ based ${ }^{\mathrm{C}}$ on data from the Langmuir probe so that $\mathrm{V}_{\text {}}$ is in the range $5000-21,000 \mathrm{~km} / \mathrm{s}$ depending on the ${ }^{\text {ion }}$ composition of the ionosphere. In this case the wave burst would arrive at the latest during the occurrence of the electron burst. Ion modes have velocities near the ion thermal velocity. For example, the electrostatic ion cyclotron mode, which propagates at frequencies above the ion gyrofrequency, has a group velocity parallel to $B$ approximately one third the ion thermal velocity (Kindel and Kennel 1971) in a single ion plasma. The most common ions at this altitude are $\mathrm{O}^{+}$and $\mathrm{H}^{+}$and based on lower hybrid resonance noise the rocket was at this time near the transition height. The thermal velocity of $\mathrm{O}^{+}$ions is of the order of $1 \mathrm{~km} / \mathrm{s}$, and of $\mathrm{H}^{+}, 4 \mathrm{~km} / \mathrm{s}$. With wave velocities of a few $\mathrm{km} / \mathrm{s}$ the velocity dispersion between electrons and waves of $8.8 \mathrm{~s}$ could be achieved if the source region was at most $40 \mathrm{~km}$ from the rocket. Thus only if the waves were ion mode waves is it possible to obtain a self-consistent relationship between the electrons and the waves. It is not possible to identify the wave mode with certainty from a comparison of the various wave components measured at the rocket but the measurements are not inconsistent with this interpretation.

If the time variation in the electron spectrum is attributed to velocity dispersion from a source at $6000 \mathrm{~km}$ distance then the wave bursts cannot be associated directly with the acceleration of the electrons because none of the possible wave modes would give the correct time delay. If the source of both wave and accelerated electrons is no more than $40 \mathrm{~km}$ away then a self-consistent interpretation is achieved with the electron spectral variation then being the result of a time variation in the acceleration.

The suggested geometrical relationship between the rocket and the source region is shown in Fig. 4. The electrons are confined by the magnetic field and can only be detected if the rocket is on the same field line as the source region. The waves, on the other hand, propagate across field lines and may be detected over a wider region. A wave burst might therefore be observed without a corresponding electron burst (point 1 against the association). Since the waves propagate over a wide range of directions they will attenuate rapidly with distance from the source. Unless the rocket is close to the source it is unlikely to detect the wave burst. Electrons on the other hand can be detected hundreds of $\mathrm{kms}$ below a source as long as the rocket is on the same field line. Furthermore if the separation becomes too large the waves would not be associated with the electrons, because the delay would be too great. Therefore it would seem to require the fortuitous occurrence of a burst source within a few $\mathrm{km}$ of the rocket for the waves and electrons to be detected together (point? against). Such associated events are likely to be rare.

Finally if the spatial relationship changes during a series of events, due for example to rocket motion, then the delay between electrons and waves will change (point 3 against).

If the association of waves and electrons is correct then it shows that: 1) the acceleration of the electrons in suprathermal bursts is accompanied by the generation of ion waves; 2) the acceleration region may be located at an altitude of $800 \mathrm{~km}$ or less; 3) the temporal duration of the burst must be given by the wave burst profile and hence is only a few seconds or less; 4) the thickness of the acceleration region parallel to $B$ must be less than the wave velocity times the burst duration, i.e. $16 \mathrm{~km}$.

Acknowledgements. We gratefully acknowledge the support of the Science Research Council for the 1976/77 High Latitude Campaign and the technical support of British Aerospace, the Appleton Laboratory and the Andoya Rocket Range.

\section{References}

Gibbons, W., Madahar, B.K. and Woolliscroft, L.J.C., Magnetospheric waves measured on high altitude Skylark rockets SL1423 and SL1424. Space Research XXI (to be published), 1980.

Johnstone, A.D. and Sojka, J.J., A beam/plasma interaction in the high altitude auroral ionosphere. Planet. Space Sci., 28, 467-474, 1980 .

Kindel, J.M. and Kennel, C.F., Topside current instabilities, J. Geophys. Res., 므, 3055-3078, 1971.

Raitt, W.J. and Sojka, J.J., Field aligned suprathermal electron fluxes below $270 \mathrm{~km}$ in the auroral zone. Planet. Space Sci., 25, 5-13, 1977.

Smith, M.J., Bryant, D.A. and Edwards, T., Pulsations in auroral electrons and positive ions, J. Atmos. Terrest. Phys., 42, 167-178, 1980 .
(Received December 16, 1980; accepted January 15, 1981.) 